\title{
The Research of Secondary Neutral Particles Spatial Distribution of Secondary Ion Mass Spectrometry
}

\author{
WANG PEI-ZHI ${ }^{1}$, BAO ZE-MIN ${ }^{2}$, LONG TAO ${ }^{3}$. \\ ${ }^{1}$ Institute of Geology Chinese Academy of Geological \\ Sciences. Beijing 102206, China. \\ wangpeizhi840923@163.com \\ ${ }^{2}$ Institute of Geology Chinese Academy of Geological \\ Sciences. Beijing 102206,China. bzmin2388@qq.com \\ ${ }^{3}$ Institute of Geology Chinese Academy of Geological \\ Sciences. Beijing 102206,China. longtao1984@163.com
}

In order to improve the sensitivity of Secondary Ion Mass Spectrometer (SIMS). Introduce femtosecond laser to ionize the secondary neutral particles which produced by primary ion bombardment. In this experiment, the primary ion of oxygen shot the surface of the sample, which was made of pure silver and copper, with $15 \mathrm{keV}$ energy and 45 angle. About $1.2 \mathrm{~mm}$ above the sample surface, the femtosecond laser was introduced and the secondary neutral particles were ionized by the former. With the impact of the extraction electrode electric field, the ion bombarded by the primary ion and the laser post-ionization ion were simultaneously introduced perpendicular to the sample surface to the independent research and development time-of-flight mass spectrometry (TOF-SIMS), and then was caught and analyzed by the Ion detector.

Experimental result shows that neutral particles have a larger percentage of the whole particles produced by primary ion. Femtosecond post-ionization techniques can improve the sensitivity of the instrument for more than seventy times, and also obtain the accurate isotope ratio. The spatial distributions of neutral particles are in concordance with MaxwellBoltzmann distribution model. The successful application of femtosecond laser post-ionization could improve the sensitivity of the SIMS, and avoid the impact of matrix effect, which could improve the SIMS accuracy of the quantitative analysis of the trace even the ultratrace element in the geologic sample. Therefore it could promote the wider use of SIMS in geologic fields such as geological dating, vertical distribution of film material, strain silicon material analysis and distribution of isotope abundance in meteorites, etc.

\section{References:}

[1] F.M. Kimock, et al. 1983. Surface Science. 124(2-3):L41L48. [2] N. Winograd, et al. 1982. Chemical Physics Letters. 88(6):581-584. [3] Bian Che-Guang, et al. 2015. Chinese Journal of Analytical Chemistry.43(8):1241-1246. 